\title{
Low-Temperature Decoherence of Qubit Coupled to Background Charges
}

\author{
Alex Grishin, Igor V. Yurkevich, and Igor V. Lerner \\ School of Physics and Astronomy, University of Birmingham, Birmingham B15 2TT, United Kingdom
}

(Dated: December 14, 2004)

\begin{abstract}
We have found an exact expression for the decoherence rate of a Josephson charge qubit coupled to fluctuating background charges. At low temperatures $T$ the decoherence rate $\Gamma$ is linear in $T$ while at high temperatures it saturates in agreement with a known classical solution which, however, reached at surprisingly high $T$. In contrast to the classical picture, impurity states spread in a wide interval of energies $(\gg T)$ may essentially contribute to $\Gamma$.

PACS numbers: 73.21.-b, 74.78.Na, 03.65.Yz
\end{abstract}

Solid state nanoscale devices provide one of the most promising routes to implementing a scalable set of controllable two-state quantum systems, qubits, based either on spin degrees of freedom of electrons in quantum dots 1] or on discrete charge quantum states in Josephson junctions 2]. An unavoidable coupling of each qubit to the environment leads to decoherence. The loss of coherence before a sufficient amount of quantum operations was performed would be the major impediment in using solid-state qubits in quantum computations.

Recent experiments on Josephson-junction (JJ) single qubits 3, 4] have demonstrated the possibility of performing hundreds of "quantum operations" (i.e. coherent oscillations between the qubit states) before environmental decoherence sets in. It is believed, however, that tens of thousands of such operations are required for quantum computation to become a reality [5] so that much longer decoherence times should be achieved experimentally. It necessitates a better theoretical understanding of realistic mechanisms of decoherence.

The most conventional way to describe environmental decoherence is based on the spin-boson models (see for reviews [6]) where the qubit interacts with environment represented as a set of harmonic oscillators with a given frequency spectrum. An alternative approach is based on identifying the dominant mechanism of decoherence in a real experimental situation and formulating the adequate model. The results obtained in such a model are not necessarily reducible to those in the spin-boson models. This turns out to be the case for decoherence in charge JJ qubits addressed in this Letter.

It is widely believed that in charge qubits $\underline{3}$ the main contribution to decoherence comes from an inevitable coupling to charge degrees of freedom, which is also responsible for $1 / f$ noise observed in such devices $7,[8]$. As charge impurities are spatially frozen as experimental temperatures $T \sim 30 \div 50 \mathrm{mK}$ [3, 7, [8], the most probable source of dynamical electromagnetic fields is impurities recharging, e.g. due to the hybridization of their electronic states with conduction electrons (in the metallic electrodes, etc.) The appropriate model similar to the conventional model of the spectral diffusion in glasses [9] is known as the spin-fluctuator model. It has already been used 10, 11, 12, 13] for a classical ("hightemperature") description of decoherence and noise in charge JJ qubits. However, in such a description contributions from impurities with energy levels outside a narrow (of order $T$ ) strip around the Fermi level of conduction electrons are exponentially suppressed. Had this been the case, decoherence from fluctuating charge impurities would hardly be seen in experiments as it is rather unlikely to find such a fluctuator coupled to the qubit in the energy strip so narrow compared to the typical (atomic) scale over which impurity levels are distributed.

In this Letter we develop a complete quantum mechanical description of decoherence due to charge fluctuators and obtain asymptotically exact expression for the decoherence rate $\Gamma(T)$ given by Eq. (12). In the classical "high- $T$ " regime it goes over to the previously obtained result [10, 11] while in the low- $T$ regime $\Gamma(T)$ decreases linearly with $T$, Eq. (15), having a nontrivial non-monotonic dependence on the coupling strength $g$. In particular, the exponential suppression of the contributions of energetically remote impurities turns out to be an artefact of the classical description. The hybridization with the conduction electrons responsible for the dynamical recharging leads also to a quantum broadening of the impurity levels which results in the contribution of (inevitably present) energetically remote impurities being suppressed only as power-law rather than exponentially, thus making it detectable and eventually dominant.

We consider a model where a charge qubit is coupled to impurities with charge states fluctuating due to hybridization with the conduction band:

$$
\begin{aligned}
& \hat{H}=\frac{\omega_{0}}{2} \hat{\sigma}_{z}-\frac{E_{J}}{2} \sigma_{x}+\hat{\sigma}_{z} \hat{V}+\hat{H}_{B}, \quad \hat{V} \equiv \frac{1}{2} \sum_{i} v_{i} \hat{d}_{i}^{\dagger} \hat{d}_{i} \\
& \hat{H}_{B}=\sum_{i} \varepsilon_{i}^{0} \hat{d}_{i}^{\dagger} \hat{d}_{i}+\sum_{i, \mathbf{k}}\left[t_{\mathbf{k} i} \hat{c}_{\mathbf{k}}^{\dagger} \hat{d}_{i}+\text { h.c. }\right]+\sum_{\mathbf{k}} \varepsilon_{\mathbf{k}} \hat{c}_{\mathbf{k}}^{\dagger} \hat{c}_{\mathbf{k}} .
\end{aligned}
$$

Here $\omega_{0}$ is the energy split and $E_{J}$ is the Josephson coupling of the two qubit states separated from the higher states by the Coulomb blockade energy $E_{c} \gg T$; both $\omega_{0}$ and $E_{J}$ are tuneable which allows one to perform quantum operations on the qubit. Each localized impurity state is characterized by its coupling to the qubit $v_{i}$ (due 
to the dipole interaction), its energy $\varepsilon_{i}$ (counted from the Fermi energy $\varepsilon_{F}$ of the conduction electrons), and its switching rate $\gamma_{i}=2 \pi \nu\left|t_{i}\right|^{2}$ ( $\nu$ is the density of states at $\varepsilon_{F}$ in the conduction band and $\left.\left|t_{i}\right|^{2}=\mathrm{Vol} \cdot\left|t_{i \mathbf{k}}\right|^{2}\right)$. All the parameters $\varepsilon_{i}^{0}, \gamma_{i}$ and $v_{i}$, vary independently in a wide interval of energies.

Solving the Heisenberg equation of motion for the full density matrix with separable initial conditions, $\hat{\rho}_{\mathrm{B}+\mathrm{Q}}(0)=\hat{\rho}(0) \otimes \hat{\rho}_{\mathrm{B}}$, where $\hat{\rho}(t)$ is the reduced density matrix of the qubit and $\hat{\rho}_{\mathrm{B}}=Z_{\mathrm{B}}^{-1} \mathrm{e}^{-\beta \hat{H}_{\mathrm{B}}}$ is the equilibrium density matrix of the bath, one writes $\hat{\rho}(t)$ in the standard formal representation (see, e.g., 11]):

$$
\hat{\rho}(t)=\left(\begin{array}{cc}
n(t) & \rho_{12}(0) \mathrm{e}^{-i \omega_{0} t} D(t) \\
\rho_{21}(0) \mathrm{e}^{i \omega_{0} t} D^{*}(t) & 1-n(t)
\end{array}\right)
$$

For the charge qubit under consideration, the operational condition is $E_{J} \ll \omega_{0}[\underline{3}]$. We are interested in decoherence only so that we restrict considerations to the 'pure dephasing' regime [10], $E_{J}=0$. In the perturbative region, small $E_{J}$ would not lead to noticeable corrections to decoherence. Although $E_{J}$ could be tuned to a large value if an operation is performed on the qubit, this should happen only for a short period of time which gives only negligible corrections to decoherence.

For $E_{J}=0$ the coupling $\sigma_{z} \hat{V}$ the diagonal elements of $\hat{\rho}$ do not evolve while the time evolution of the offdiagonal elements of $\hat{\rho}$ can be represented as

$$
D(t)=\left\langle\mathrm{e}^{i\left(\hat{H}_{B}+\hat{V}\right) t} \mathrm{e}^{-i\left(\hat{H}_{B}-\hat{V}\right) t}\right\rangle_{\mathrm{B}}
$$

where $\langle\ldots\rangle_{\mathrm{B}}$ is the average with the Gibbs density matrix of the bath, $\hat{\rho}_{\mathrm{B}}$. Expressions of this sort can be exactly calculated in certain problems, e.g. the orthogonality catastrophe 14] and full counting statistics [15] in $1 D$, in techniques which are always problem-specific. In present considerations, we employ the linked-cluster expansion within the Keldysh formalism (similar to that used in [16] for bosonic environment) to find the decoherence rate $\Gamma$ defined by

$$
\Gamma(T)=-\lim _{t \rightarrow \infty} t^{-1} \ln |D(t)| .
$$

In order to calculate $\Gamma$, we represent $D(t)$ in Eq. (3) as the following functional integral over the Grassmann fields defined on the standard Keldysh contour $c_{\mathrm{K}}$ [17]:

$$
D(t)=\int \frac{\mathcal{D} \bar{d} \mathcal{D} d \mathcal{D} \bar{c} \mathcal{D} c}{\mathcal{Z}} \exp \left[i \int_{c_{\mathrm{K}}} \mathrm{d} t^{\prime}\left(\sum_{i j} S_{i j}+\sum_{\mathbf{k}} S_{\mathbf{k}}\right)\right],
$$

where the action densities are given by

$$
\begin{aligned}
& S_{i j}=\bar{d}_{j}\left(t^{\prime}\right)\left(i \partial_{t^{\prime}}-\varepsilon_{j}^{0}+\frac{v_{j}\left(t^{\prime}\right)}{2}\right) d_{j}\left(t^{\prime}\right) \delta_{i j} \\
& S_{\mathbf{k}}=\bar{c}_{\mathbf{k}}\left(t^{\prime}\right)\left(i \partial_{t^{\prime}}-\varepsilon_{\mathbf{k}}\right) c_{\mathbf{k}}\left(t^{\prime}\right)-\sum_{i}\left(t_{\mathbf{k} i} \bar{c}_{\mathbf{k}}\left(t^{\prime}\right) d_{i}\left(t^{\prime}\right)+\text { h.c. }\right) .
\end{aligned}
$$

The field $v_{j}\left(t^{\prime}\right)= \pm v_{j}$ for $0 \leq t^{\prime} \leq t$, with ' + ' sign on the upper and '-' sign on the lower branch of the Keldysh contour, and vanishes for $t^{\prime}<0$ or $t^{\prime}>t$. The normalization $\mathcal{Z}$ is defined as the same functional integral but with $v_{j} \equiv 0$. The integration over the conduction electron fields $\bar{c}_{\mathbf{k}}, c_{\mathbf{k}}$ reduces the action (5) to the impurity term $S_{i j}$ with the mass operator

$$
\Sigma_{i j}\left(t^{\prime}, t^{\prime \prime}\right)=\sum_{\mathbf{k}} t_{\mathbf{k} i} t_{\mathbf{k} j}^{*} g_{\mathbf{k}}\left(t^{\prime}, t^{\prime \prime}\right),
$$

where $g_{\mathbf{k}}\left(t^{\prime}, t^{\prime \prime}\right)$ is the conduction electron Green function obeying the equation $\left(i \partial_{t^{\prime}}-\varepsilon_{\mathbf{k}}\right) g_{\mathbf{k}}\left(t^{\prime}, t^{\prime \prime}\right)=\delta\left(t^{\prime}, t^{\prime \prime}\right)$ with the delta function defined on the Keldysh contour. Now the integration over the fields $\bar{d}_{i}$ and $d_{i}$ reduces $D(t)$, Eq. (3), to the appropriate matrix determinant thus yielding the following formal result for $\Gamma, \mathrm{Eq}$. (4):

$$
\Gamma(T)=-\Re \mathrm{e} \lim _{t \rightarrow \infty} t^{-1} \operatorname{Tr} \ln \left[1+\frac{\hat{v}}{2} \hat{G}\right]
$$

where $\hat{G}$ obeys $\left(i \partial_{t^{\prime}}-\hat{\varepsilon}^{0}-\widehat{\Sigma}\right) \hat{G}=\hat{I}$, and Tr implies an integration over times along the Keldysh contour and a summation over indices labelling the fluctuators. The dependence on the running time $t$ above is via $v_{j}(t)$ defined after Eq. (57). The long- $t$ limit in Eq. (7) can be found by expanding $\operatorname{Tr} \ln$ into power series in $\hat{v} \hat{G}$. The $n^{\text {th }}$ order term of the expansion is a multiple integral over $n$ time variables, each running along that part of $c_{\mathrm{K}}$ where $v \neq 0$. As usual [17], we represent each integrand via the Keldysh matrices

$$
\hat{\mathcal{G}}=\left(\begin{array}{cc}
\hat{G}^{R} & \hat{G}^{K} \\
0 & \hat{G}^{A}
\end{array}\right)
$$

thus reducing each integration over time to that from 0 to $t$ (with $v \rightarrow v \tau_{x}$, where $\tau_{x}$ is the Pauli matrix in the Keldysh space). Due to the time translation invariance, each $\hat{G}$ depends only on the difference of its time arguments. Then the $n^{\text {th }}$ order integrand depends on $n-1$ differences in times, while the integration over the last time variable produces the overall factor of $t$. The remaining integrals in time can be extended to the entire axis as all the Green functions exponentially decay in time with the time constants $\gamma_{i}^{-1}$ (or $T^{-1}$ for the real part of the Keldysh component $G^{\mathrm{K}}$ ). Then the integral has a convolution structure in time and, upon a Fourier transform, it finally reduces to the integral of $t\left[\frac{1}{2} \hat{v} \mathcal{G}(\omega)\right]^{n}$ over $\omega$. Since the coefficients of the expansion were not affected by the Fourier transform, the re-summing the series restores the logarithm and Eq. (17) reduces to

$$
\Gamma=-\Re \mathrm{e} \int_{-\infty}^{+\infty} \frac{\mathrm{d} \omega}{2 \pi} \operatorname{tr} \ln \left[1+\frac{v}{2} \hat{G}^{\mathrm{K}}-\frac{v}{2} \hat{G}^{\mathrm{R}} \frac{v}{2} \hat{G}^{\mathrm{A}}\right]
$$

where the trace of $\ln \left[1+\frac{\hat{v}}{2} \tau_{x} \hat{\mathcal{G}}(\omega)\right]$ in the space of matrices (8) has been explicitly taken and tr refers only to the fluctuator matrix indices. 
It follows from Eq. (9) that $\Gamma_{0} \equiv \Gamma(T=0)$ vanishes as expected. Indeed, at $T=0$ one uses [17] $G^{\mathrm{K}}(\omega)=$ $\left[G^{\mathrm{R}}(\omega)-G^{\mathrm{A}}(\omega)\right] \operatorname{sgn} \omega$ to find

$$
\Gamma_{0}=-\Re e \int_{-\infty}^{+\infty} \frac{\mathrm{d} \omega}{2 \pi} \operatorname{tr} \ln \left[\left(1+\frac{\hat{v}}{2} \hat{G}^{\mathrm{R}}\right)\left(1-\frac{\hat{v}}{2} \hat{G}^{\mathrm{A}}\right)\right]=0,
$$

since the first order of the expansion is imaginary, while the higher orders vanish upon the integration as all the poles are in the upper (lower) $\omega$ half-plane.

A further simplification is possible for a typical situation when distances between the fluctuators are larger than the Fermi wavelength $k_{\mathrm{F}}^{-1}$ so that the hybridization is local, $t_{\mathbf{k} i}=t_{i} V^{-1 / 2} \mathrm{e}^{i \mathbf{k r}_{i}}$. Then Eq. (6) reduces to $\Sigma_{i j}=t_{i} t_{j}^{*} g\left(\mathbf{r}_{i}-\mathbf{r}_{j}, t^{\prime}-t^{\prime \prime}\right)$. Since $g(\mathbf{r})$ (the Fourier transform of the conduction electron Green function $g_{\mathbf{k}}$ ) oscillates at $k_{\mathrm{F}}^{-1}$, off-diagonal matrix elements of $\widehat{\Sigma}$ vanish upon the integration (neglecting small interference corrections) making $\hat{G}$ in Eq. (17) diagonal in the fluctuator indices, $\hat{G}=G_{j} \delta_{i j}$ (such a diagonal form was assumed in 10. by choosing independent conduction bands for each impurity as in [18].) Here

$$
G_{j}^{\mathrm{R} / A}(\omega)=\left(\omega-\varepsilon_{j} \pm i \frac{\gamma_{j}}{2}\right)^{-1},
$$

where $\gamma_{j} \equiv 2 \Im \mathrm{m} \Sigma_{j j}^{\mathrm{R}}=2 \pi \nu\left|t_{j}\right|^{2}$ and $\varepsilon_{j}=\varepsilon_{j}^{0}+\Re \mathrm{e} \Sigma_{j j}$ is the fluctuator energy (counted from $\varepsilon_{\mathrm{F}}$ ) renormalized by the hybridization. The Keldysh component of $\hat{\mathcal{G}}$ is given by [17] $G^{\mathrm{K}}(\omega)=\left[G^{\mathrm{R}}(\omega)-G_{j}^{\mathrm{A}}(\omega)\right]\left[1-2 n_{\mathrm{F}}(\omega)\right]$, where $n_{\mathrm{F}}(\omega)$ is the Fermi factor with $\omega$ counted from $\varepsilon_{\mathrm{F}}$.

Then $\Gamma$ in Eq. (4) reduces to a sum of the individual fluctuator contributions, $\Gamma(T)=\sum_{j} \Gamma_{j}(T)$. Substituting $\hat{G}_{j}$ of Eq. (11) into Eq. (9), subtracting the identically zero expression (10) for $\Gamma_{0}$ (to improve the integral convergency) and taking the real part of the resulting expression, we obtain the following contribution of a single fluctuator at energy $\varepsilon_{j} \equiv \varepsilon$ to the decoherence rate:

$$
\Gamma_{\varepsilon}(T)=-\int_{-\infty}^{+\infty} \frac{d \omega}{4 \pi} \ln \left\{1-\frac{4 n_{\mathrm{F}}(\omega)\left[1-n_{\mathrm{F}}(\omega)\right]}{1+\left[\lambda_{\varepsilon}^{-1}(\omega)-\frac{1}{2} g\right]^{2}}\right\} .
$$

Here we suppressed the index $j$ and introduced dimensionless coupling $g \equiv v / \gamma$ of the qubit and the fluctuator with the dimensionless density of states $\lambda_{\varepsilon}(\omega) \equiv \pi v \nu_{\varepsilon}(\omega)$ broadened around the energy $\varepsilon$ by the hybridization:

$$
\nu_{\varepsilon}(\omega)=-\frac{1}{\pi} \Im \mathrm{m} G_{\varepsilon}^{\mathrm{R}}(\omega)=\frac{1}{2 \pi} \frac{\gamma}{(\omega-\varepsilon)^{2}+\gamma^{2} / 4} .
$$

The temperature dependence of the decoherence rate (12) is presented in Fig. 1 for the strong $(g=100)$ and weak $(g=0.2)$ coupling to a single fluctuator with $\nu_{\varepsilon}(\omega)$ centered at $\widetilde{\varepsilon} \equiv 2 \varepsilon / \gamma=3$ (or $\varepsilon=0$, insert). In the high- $T$ limit, $\Gamma$ saturates at the following $\varepsilon$-independent value:

$$
\Gamma(\infty)=\frac{\gamma}{2}\left[1-\theta(1-g) \sqrt{1-g^{2}}\right] .
$$

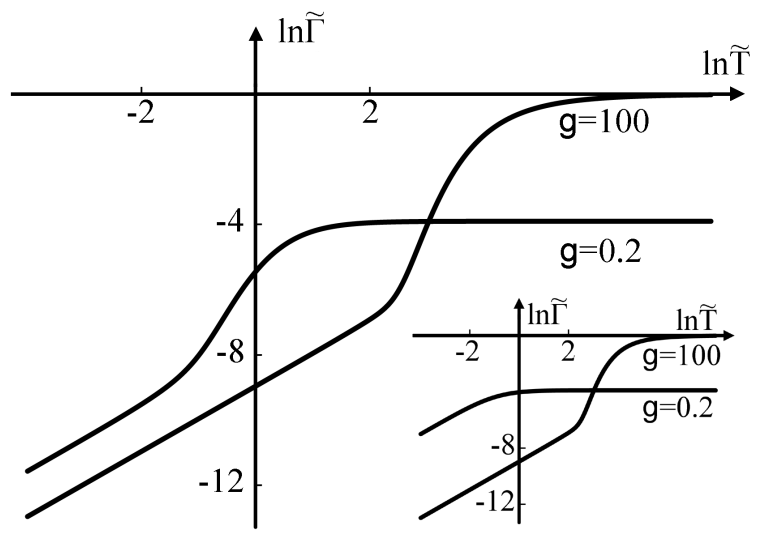

FIG. 1: Dependence of the decoherence rate on a temperature, Eq. (12), for strong and week coupling: the main picture is for a fluctuator with $\nu_{\varepsilon}(\omega)$ centered at $\widetilde{\varepsilon}=3$, the insert for $\widetilde{\varepsilon}=0$; here $\widetilde{T}, \widetilde{\Gamma}, \widetilde{\varepsilon}$ are measured in units of $\gamma / 2$.

Although it coincides with the results [10, 11] of the classical "high- $T$ " description, it follows from the exact expression (12) only at $T \gg \max \left\{\left|\varepsilon_{ \pm}\right|, \gamma\right\}$, where $\varepsilon_{ \pm} \equiv \varepsilon \pm(1 / 2) \sqrt{v^{2}-\gamma^{2}}$. Thus, for a fluctuator with either relatively large $\varepsilon$ or large $v$ (and thus $\left|\varepsilon_{ \pm}\right|$), the classical regime (14) is never reached. Then $\Gamma$ is described at any temperature by the "low- $T$ " asymptotics

$$
\Gamma(T)=\frac{T}{\pi} \arctan ^{2}\left(\frac{2 g}{\widetilde{\varepsilon}^{2}-g^{2}+1}\right),
$$

which follows for any $\varepsilon$ from Eq. (12) either for $T \ll$ $\gamma$ or for $T \ll \min \left\{\left|\varepsilon_{ \pm}\right|\right\}$. This means that the result (14) of the classical description 10, 11] is applicable only for a fluctuator with $\nu_{\varepsilon}(\omega)$ centered near the Fermi level provided that $T \gg \max (v, \gamma)$.

Note that a crossover between the asymptotics (15) and (14) is relatively sharp when $A \equiv \max (g, \widetilde{\varepsilon}) \gg 1$ : $\Gamma(T)$ changes exponentially fast,

$$
\Gamma(T) \sim \Gamma_{\infty} \exp (-A \gamma / 2 T),
$$

in a logarithmically narrow interval, $A / \ln A \lesssim T / \gamma \lesssim A$.

Although a linear in $T$ behaviour similar to that in Eq. (15) would also follow from the spin-boson models with the ohmic spectral function, only a full quantum treatment of a microscopic model, like that in Eq. (11), can result in a nontrivial $T$-dependence depicted in Fig. 1.

One specific and surprising feature of the model (11) is a non-monotonic dependence of the decoherence rate $\Gamma$ on the coupling strength $g$ : at low $T$ a contribution of weakly coupled fluctuators can be orders of magnitude higher than that of strongly coupled ones, as seen in Fig. 1. Such a non-monotonic dependence is depicted in Fig. 2 for the fluctuator centered at $\widetilde{\varepsilon}=3$. At any finite temperature $\Gamma$ as a function of $g$ has a maximum with a cusp at $g_{0}=$ $\left(1+\widetilde{\varepsilon}^{2}\right)^{1 / 2}$. Only at very high $T(\widetilde{T}=100$ in Fig. 2) 


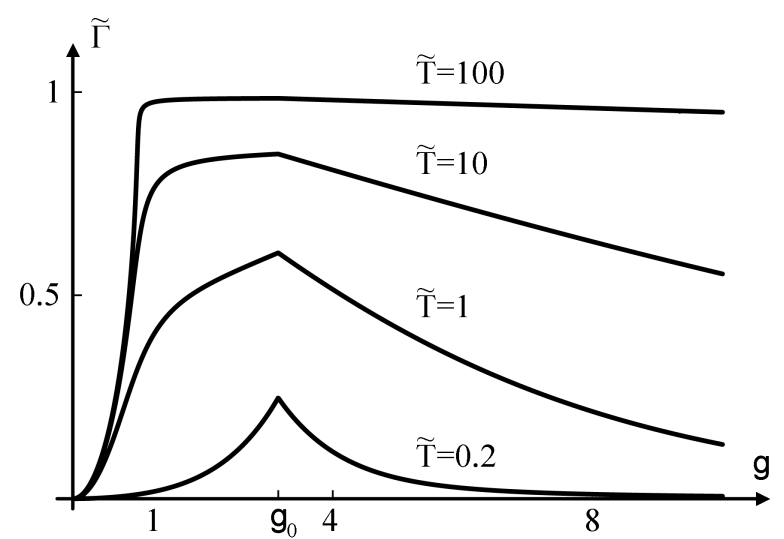

FIG. 2: Non-monotonic dependence of the decoherence rate on the coupling strength at different temperatures for $\widetilde{\varepsilon}=3$.

the cusp is smeared out and $\Gamma$ practically saturates at $\Gamma(\infty)=\gamma / 2$ as in the classical limit, Eq. (14).

This surprising suppression is due to an effective spit in energy of fluctuators strongly coupled to the qubit. If the qubit were in one of its eigenstates, the fluctuator energy in the absence of the hybridization would be split as $\varepsilon \pm v / 2$, Eq. (11). Allowing for the hybridization, such a split to the energies $\varepsilon_{ \pm} \equiv \varepsilon \pm(1 / 2) \sqrt{v^{2}-\gamma^{2}}$ occurs only for $g>1$, i.e. when the coupling $v$ exceeds the width $\gamma$ of the hybridized fluctuator Eq. (13). Then the decoherence of the qubit in the mixed state, Eq. (2), is effectively contributed from two peaks $\varepsilon_{ \pm}$(indeed, the integrand in Eq. (12) has the two peaks broadened by the hybridization at $\omega=\varepsilon_{ \pm}$, besides the exponentially narrow peak at $\omega=0)$. Thus, $\Gamma$ increases with $g$ at $\varepsilon=0$ until $v$ reaches $\gamma$; a further increase in $g$ pushes the peaks away from the Fermi energy, suppressing the hybridization and thus the switching rate i.e. effectively freezing the charge states. For the arbitrary $\varepsilon$, the maximum in $\Gamma$ is reached when one of the peaks at $\varepsilon_{ \pm}$coincides with the Fermi energy.

The results described by Eqs. (12) - 15) and the subsequent discussion refer to the case when there are only a few fluctuators, so that they can be considered separately and their decoherence rates could be simply added. If the fluctuators are dense, one needs to average over the relevant parameters, which is their energies, coupling constants and switching rates. The averaging, e.g., over the energy positions $\varepsilon_{j}$, spread within an interval $E$, would lead to $\Gamma(T) \propto T$ as long as $T \ll E$. The reason is that the number of effective high- $T$ fluctuators, whose contribution is described by Eq. (14), would be proportional to $T$ while the contribution of each of low- $T$ fluctuators would be linear in $T$, Eq. (15).

However, as the decoherence rates due to individual fluctuators are hugely spread, as illustrated in Fig. 1, the effective fluctuators are hardly dense as requirements for the effectiveness are rather restrictive. Firstly, the fluc- tuator must be not far from metallic electrodes to be hybridized with conduction electrons. Secondly, the peaks at $\varepsilon_{ \pm}$should be within a few $\gamma$ 's around the Fermi energy. This brings further geometrical restrictions essentially reducing the number of potentially relevant defects so that only relatively few fluctuators are likely to contribute to decoherence in a typical experimental setup [3].

We thank B. L. Altshuler, R. Fazio, J. M. F. Gunn and R. A. Smith for useful comments. I.V.L. acknowledges the kind hospitality extended to him at the final stage of this work at Princeton University and NEC Laboratories America. This work was supported by the EPSRC grant GR/R95432 and in part by DARPA.

[1] D. Loss and D. P. DiVincenzo, Phys. Rev. A. 57, 120 (1998); D. P. DiVincenzo, Science 270, 255 (1995).

[2] A. Shnirman, G. Schön, and Z. Hermon, Phys. Rev. Lett. 79, 2371 (1997); Y. Makhlin, G. Schön, and A. Shnirman, Nature 398, 305 (1999); Rev. Mod. Phys. 73, 357 (2001).

[3] Y. Nakamura, Y. A. Pashkin, and J. S. Tsai, Nature 398, 786 (1999); Y. A. Pashkin et al., Nature 421, 823 (2003).

[4] C. H. van der Wal, A. C. J. ter Haar, F. K. Wilhelm, R. N. Schouten, C. J. P. M. Harmans, T. P. Orlando, S. Lloyd, and J. E. Mooij, Science 290, 773 (2000); D. Vion, A. Aassime, A. Cottet, P. Joyez., H. Pothier, C. Urbina, D. Esteve, and M. H. Devoret, ibid 296, 886 (2002); Y. Yu, S. Han, X. Chu, S.-I Chu, and Z. Wang, ibid, 889 (2002).

[5] D. P. DiVincenzo, and D. Loss, J. Magn. Magn. Mater. 200, 202 (1999); Phys. Rev. B. 71, 035318 (2005).

[6] A. J. Leggett, S. Chakravarty, A. T. Dorsey, M. P. A. Fisher, A. Garg, and W. Zwerger, Rev. Mod. Phys. 59, 1 (1987).

[7] A. B. Zorin, F.-J. Ahlers, J. Niemeyer, T. Weimann, H. Wolf, V. A. Krupenin, and S. V. Lotkhov, Phys. Rev. B. 53, 13682 (1996).

[8] Y. Nakamura, Y. A. Pashkin, T. Yamamoto, and J. S. Tsai, Phys. Rev. Lett. 88, 047901 (2002).

[9] J. R. Klauder and P. W. Anderson, Phys. Rev. 125, 912 (1962); J. L. Black and B. I. Halperin, Phys. Rev. B. 16, 2879 (1977); B. D. Laikhtman, ibid 31, 490 (1985).

[10] E. Paladino, L. Faoro, G. Falci, and R. Fazio, Phys. Rev. Lett. 88, 228304 (2002).

[11] Y. M. Galperin, B. L. Altshuler, and D. V. Shantsev, cond-mat/0312490 (2003).

[12] E. Paladino, A. Mastellone, A. D'Arrigo, and G. Falci, cond-mat/0407484 (2004).

[13] L. Faoro, J. Bergli, B. L. Altshuler, and Y. M. Galperin, cond-mat/0411425 (2004).

[14] G. D. Mahan, Many-Particle Physics, Plenum Press, London (1990).

[15] L. S. Levitov, H. Lee, G. B. Lesovik, J. Math. Phys., 37, 4845 (1996).

[16] Y. Makhlin, A. Shnirman, Phys. Rev. Lett. 92, 178301 (2004).

[17] J. Rammer and H. Smith, Rev. Mod. Phys. 58, 323 (1986).

[18] R. Bauernschmitt and Y. V. Nazarov, Phys. Rev. B. 47, R9997 (1993). 